\title{
Gate-tunable bandgap in bilayer graphene
}

\author{
L.A. Falkovsky ${ }^{1,2}$ \\ ${ }^{1}$ L.D. Landau Institute for Theoretical Physics, Moscow 117334, Russia \\ ${ }^{2}$ Institute of the High Pressure Physics, Troitsk 142190, Russia
}

(Dated: October 30, 2018)

\begin{abstract}
The tight-binding model of bilayer graphene is used to find the gap between the conduction and valence bands, as a function of both the gate voltage and as the doping by donors or acceptors. The total Hartree energy is minimized and the equation for the gap is obtained. This equation for the ratio of the gap to the chemical potential is determined only by the screening constant. Thus the gap is strictly proportional to the gate voltage or the carrier concentration in the absence of donors or acceptors. In the opposite case, where the donors or acceptors are present, the gap demonstrates the asymmetrical behavior on the electron and hole sides of the gate bias. A comparison with experimental data obtained by Kuzmenko et al demonstrates the good agreement.
\end{abstract}

PACS numbers: 73.20.At, 73.21.Ac, 73.43.-f, 81.05.Uw

\section{INTRODUCTION}

Bilayer graphene has attracted much interest partly due to the opening of a tunable gap in its electronic spectrum by an external electrostatic field. Such a phenomenon was predicted in Refs. [1, 2] and can be observed in optical studies controlled by applying a gate bias [3, 4, 5, 6, 7, 8, 9, 10]. In Refs. [11, 12], within the self-consistent Hartree approximation, the gap was derived as a near-linear function of the carrier concentration injected in the bilayer by the gate bias. Recently, this problem was numerically considered in Ref. 13] using the density functional theory (DFT) and including the external charge doping involved with impurities. The DFT calculation gives the gap which is roughly half of the gap obtained in the Hartree approximation. This disagreement was explained in Ref. [13] as a result of both the inter- and intralayer correlations.

In this work, we study this problem within the same Hartree approximation as in Refs. 11, 12], but including the effect of external doping. We calculate the carrier concentration on both sides of the bilayer considering the case, where the carrier concentration in the bilayer is less than $10^{13} \mathrm{~cm}^{-2}$. Then, we minimize the total energy of the system and find self-consistently both the chemical potential and the gap induced by the gate bias. Our results completely differ from those obtained in Refs. 11, 12], where the external doping is disregarded. In the presence of dopants, the dependence of the gap on the carrier concentration, i.e., on the gate voltage, exhibits an asymmetry at the electron and hole sides of the gate bias.

\section{TIGHT-BINDING MODEL OF BILAYER GRAPHENE}

The graphene bilayer lattice is shown in Fig. 1. Atoms in one layer, i. e., $a$ and $b$ in the unit cell, are connected by solid lines, and in the other layer, e.g., $a_{1}$ and $b_{1}$, by the dashed lines. The atom $a\left(a_{1}\right)$ differs from $b\left(b_{1}\right)$ because it has a neighbor just below in the adjacent layer, whereas the atom $b\left(b_{1}\right)$ does not.

Let us recall the main results of the SlonchewskiWeiss-McClure model [14, 15]. In the tight-binding model, the Bloch functions of the bilayer are written as

$$
\begin{array}{r}
\psi_{a}=\frac{1}{\sqrt{N}} \sum_{j} e^{i \mathbf{k a}_{j}} \psi_{0}\left(\mathbf{a}_{j}-\mathbf{r}\right) \\
\psi_{b}=\frac{1}{\sqrt{N}} \sum_{j} e^{i \mathbf{k a}_{j}} \psi_{0}\left(\mathbf{a}_{j}+\mathbf{a}-\mathbf{r}\right) \\
\psi_{a 1}=\frac{1}{\sqrt{N}} \sum_{j} e^{i \mathbf{k a}_{j}} \psi_{0}\left(\mathbf{a}_{j}+\mathbf{c}-\mathbf{r}\right) \\
\psi_{b 1}=\frac{1}{\sqrt{N}} \sum_{j} e^{i \mathbf{k a}_{j}} \psi_{0}\left(\mathbf{a}_{j}+\mathbf{c}+\mathbf{a}-\mathbf{r}\right),
\end{array}
$$

where the sums are taken over the lattice vectors $\mathbf{a}_{j}$ and $N$ is the number of unit cells. Vectors a and c connect the nearest atoms in the layer and in the neighbor layers, correspondingly.

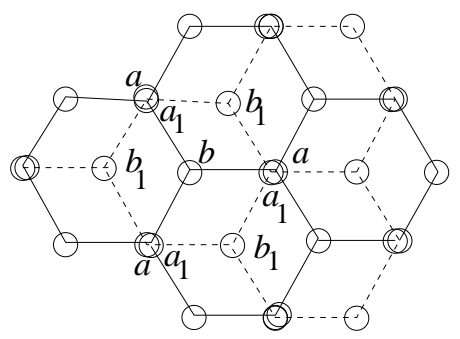

FIG. 1: Bilayer lattice

For the nearest neighbors, the effective Hamiltonian in 
the space of the functions (11) can be written as

$$
H(\mathbf{k})=\left(\begin{array}{cccc}
U+\Delta & \gamma_{0} f^{*} & \gamma_{1} & \gamma_{4} f \\
\gamma_{0} f & U-\Delta & \gamma_{4} f & \gamma_{3} f^{*} \\
\gamma_{1} & \gamma_{4} f^{*} & -U+\Delta & \gamma_{0} f \\
\gamma_{4} f^{*} & \gamma_{3} f & \gamma_{0} f^{*} & -U-\Delta
\end{array}\right)
$$

where $f=\gamma_{0}\left[e^{i k_{x} a}+2 e^{-i k_{x} a / 2} \cos \left(k_{y} a \sqrt{3} / 2\right)\right]$. The values of hopping integrals $\gamma_{0}, \gamma_{1}, \gamma_{3}, \gamma_{4}$, and $\Delta$ are given in the Table I. The largest of them, $\gamma_{0}$, determines the band

TABLE I: Parameter values of the electron spectrum in $\mathrm{eV}$.

\begin{tabular}{|c|c|c|}
\hline \hline Parameter & Experiment [10] & DFT calculation [16] \\
\hline$\gamma_{0}$ & $3.16 \pm 0.3$ & $2.598 \pm 0.015$ \\
\hline$\gamma_{1}$ & $0.381 \pm 0.003$ & $0.34 \pm 0.02$ \\
\hline$\gamma_{3}$ & $0.38 \pm 0.06$ & $0.32 \pm 0.02$ \\
\hline$\gamma_{4}$ & $0.14 \pm 0.03$ & $0.177 \pm 0.025$ \\
\hline$\Delta$ & $0.022 \pm 0.003$ & $0.024 \pm 0.01$ \\
\hline \hline
\end{tabular}

dispersion near the $K$ point in the Brillouin zone where the matrix element $\gamma_{0} f$ can be expanded as

$$
\gamma_{0} f=v\left(i k_{x}-k_{y}\right),
$$

with a velocity parameter $v=3 \gamma_{0} a / 2$. The parameters $\gamma_{3}$ and $\gamma_{4}$ giving a correction to the dispersion are less than $\gamma_{0}$ by a factor of 10 . The parameters $\gamma_{1}$ and $\Delta$ result in the position of levels at $K$, but $\Delta$ is much less than $\gamma_{1}$. There is in addition the parameter $U$ induced by the gate voltage and associated with the asymmetry of two layers in the external electrostatic field. This parameter presents the potential energy $-e d E$ between two layers, where $d$ is the interlayer distance and $E$ is electric field induced both by the gate voltage and the external dopants in the bilayer.

The parameter $U$ as well as the chemical potential $\mu$ should be self-consistently calculated for the given gate voltage. For this purpose, we can held only the parameters $\gamma_{0}$ and $\gamma_{1}$, neglecting the small effect of $\gamma_{3}, \gamma_{4}$, and $\Delta$ on the gap $U$. In this approximation, the effective Hamiltonian can be written in the simple form

$$
H(\mathbf{k})=\left(\begin{array}{cccc}
U & v k_{+} & \gamma_{1} & 0 \\
v k_{-} & U & 0 & 0 \\
\gamma_{1} & 0 & -U & v k_{-} \\
0 & 0 & v k_{+} & -U
\end{array}\right),
$$

where $k_{ \pm}=\mp i k_{x}-k_{y}$ in the vicinity of the $K$ points.

The Hamiltonian gives four energy bands:

$$
\begin{aligned}
& \varepsilon_{1,4}(q)= \pm\left(\frac{\gamma_{1}^{2}}{2}+U^{2}+q^{2}+W\right)^{1 / 2} \\
& \varepsilon_{2,3}(q)= \pm\left(\frac{\gamma_{1}^{2}}{2}+U^{2}+q^{2}-W\right)^{1 / 2}
\end{aligned}
$$

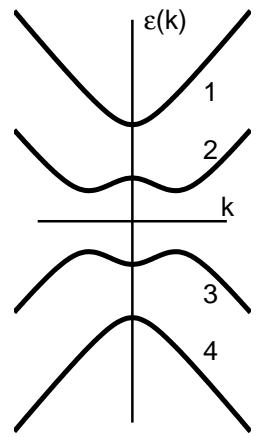

FIG. 2: Band structure of bilayer.

where

$$
W=\left(\frac{\gamma_{1}^{4}}{4}+\left(\gamma_{1}^{2}+4 U^{2}\right) q^{2}\right)^{1 / 2}
$$

and we denote $q^{2}=(v k)^{2}$.

The band structure is shown in Fig. 22, The minimal value of the upper energy $\varepsilon_{1}$ is $\sqrt{U^{2}+\gamma_{1}^{2}}$. The $\varepsilon_{2}$ band takes the maximal value $|U|$ at $q=0$ and the minimal value $\tilde{U}=\gamma_{1}|U| / \sqrt{\gamma_{1}^{2}+4 U^{2}}$ at $q^{2}=2 U^{2}\left(\gamma_{1}^{2}+\right.$ $\left.2 U^{2}\right) /\left(\gamma_{1}^{2}+4 U^{2}\right)$. Because the value of $U$ is much less than $\gamma_{1}$, the distinction between $U$ and $\tilde{U}$ is small and the gap between the bands $\varepsilon_{2}$ and $\varepsilon_{3}$ takes approximately the value $2|U|$.

\section{EIGENFUNCTIONS AND CARRIER CONCENTRATION}

The four eigenfunctions $\mathbf{C}$ corresponding with eigenvalues (44) of the Hamiltonian (3) read

$$
\mathbf{C}=\frac{1}{C}\left(\begin{array}{c}
\left(U-\varepsilon_{n}\right)\left[\left(\varepsilon_{n}+U\right)^{2}-q^{2}\right] \\
-q_{-}\left[\left(\varepsilon_{n}+U\right)^{2}-q^{2}\right] \\
\gamma_{1}\left(U^{2}-\varepsilon_{n}^{2}\right) \\
\gamma_{1} q_{+}\left(U-\varepsilon_{n}\right)
\end{array}\right),
$$

where the $\mathbf{C}$ norm squared is

$$
\begin{array}{r}
C^{2}=\left[\left(\varepsilon_{n}+U\right)^{2}-q^{2}\right]^{2}\left[\left(\varepsilon_{n}-U\right)^{2}+q^{2}\right] \\
+\gamma_{1}^{2}\left(\varepsilon_{n}-U\right)^{2}\left[\left(\varepsilon_{n}+U\right)^{2}+q^{2}\right] .
\end{array}
$$

As seen from Eqs. (10), the probability $p_{1}$ to find an electron, for instance, on the first layer is

$$
p_{1}=\left|C_{1}\right|^{2}+\left|C_{2}\right|^{2},
$$

where the subscript $C_{i}$ numerates the elements of the column (5).

We assume, that carriers occupy only the bands $\varepsilon_{2,3}$, so the chemical potential $\mu$ and the gap $2|U|$ are less 
than the distance between the bands $\varepsilon_{1}$ and $\varepsilon_{2}$, i. e., $(|\mu|, 2|U|)<\gamma_{1}$. The electron dispersion for the $\varepsilon_{2,3}$ bands can be expanded in powers of $q^{2}$ :

$$
\varepsilon_{n}^{2}(q)=U^{2}-4 \frac{U^{2}}{\gamma_{1}^{2}} q^{2}+\frac{q^{4}}{\gamma_{1}^{2}},
$$

where $n=2$ stands for the electron conductivity and $n=3$ for the hole conductivity. Then, for $q^{2} \gg 4 U^{2}$, we can omit here the second term and use the simple relations

$$
q^{2}=\gamma_{1}\left(\varepsilon_{n}^{2}-U^{2}\right)^{1 / 2}
$$

neglecting the small effect of the "mexican hat".

Keeping only the leading terms, one can find with the help of Eq. (5), that the probabilities $p_{1,2}$ to find an electron on the layers are proportional

$$
\begin{array}{r}
p_{1}=\left|C_{1}\right|^{2}+\left|C_{2}\right|^{2} \propto q^{6}=\gamma_{1}^{3}\left(\varepsilon_{n}^{2}-U^{2}\right)^{3 / 2}, \\
p_{2}=\left|C_{3}\right|^{2}+\left|C_{4}\right|^{2} \propto q^{2} \gamma_{1}^{2}\left(U-\varepsilon_{n}\right)^{2} \\
=\gamma_{1}^{3}\left(\varepsilon_{n}^{2}-U^{2}\right)^{1 / 2}\left(U-\varepsilon_{n}\right)^{2} .
\end{array}
$$

Therefore, the normalized probability to find an electron, for instance, on the first layer can be written as

$$
\begin{array}{r}
p_{1}=\frac{\left(\varepsilon_{n}^{2}-U^{2}\right)^{3 / 2}}{\left(\varepsilon_{n}^{2}-U^{2}\right)^{3 / 2}+\left(\varepsilon_{n}^{2}-U^{2}\right)^{1 / 2}\left(U-\varepsilon_{n}\right)} \\
=\left(\varepsilon_{n}+U\right) / 2 \varepsilon_{n} .
\end{array}
$$

Within the approximation (77)-(8), many observable effects can be analytically evaluated for the intermediate carrier concentration, $4 U^{2} \ll \gamma_{1} \sqrt{\mu^{2}-U^{2}} \ll \gamma_{1}^{2}$.

At zero temperature, the carrier concentration on the sides of the bilayer is found with the help of Eq. (8) as

$$
\begin{array}{r}
n_{1,2}=\frac{2}{\pi \hbar^{2} v^{2}} \int p_{1,2} q d q \\
=\frac{n_{0} U}{2 \gamma_{1}}\left[\sqrt{x^{2}-1} \pm \ln \left(x+\sqrt{x^{2}-1}\right],\right.
\end{array}
$$

where the limits of integration are $q=0$ and the chemical potential $\mu$ and we set

$$
\begin{array}{r}
n_{0}=\gamma_{1}^{2} / \pi \hbar^{2} v^{2}=1.03 \times 10^{13} \mathrm{~cm}^{-2}, \\
x=|\mu / U| .
\end{array}
$$

For the total carrier concentration $n$ in the bilayer, we obtain

$$
n=\frac{\gamma_{1}}{\pi \hbar^{2} v^{2}} \sqrt{\mu^{2}-U^{2}}=\frac{n_{0} U}{\gamma_{1}} \sqrt{x^{2}-1} .
$$

\section{MINIMIZATION OF THE TOTAL ENERGY}

In order to find the chemical potential $\mu$ and the gap $2|U|$ at the given gate voltage

$$
e V_{g}=-e d E-e d_{w} E_{w},
$$

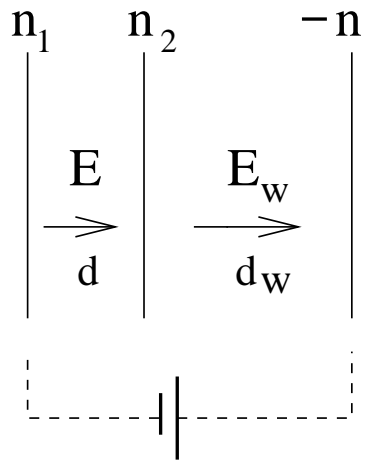

FIG. 3: Electrostatic model; $d$ is the interlayer distance, $d_{w}$ is the wafer thickness.

we minimize the total energy containing both the energy $V^{(c)}$ of the carriers and the energy $V^{(f)}$ of the electrostatic field. Within the Hartree approximation, when no electron correlations are taken into account, the filled bands do not contribute into the energy of the system, because the electron charge of the filled bands is compensated by the ion charge and this energy have to be considered as the ground state energy. The excitation energy is owes its origin to the carriers in unfilled bands. Electrons in the $\varepsilon_{2}$ band or holes in the $\varepsilon_{3}$ band contribute in the total energy of the system the energy

$$
\begin{array}{r}
V^{(c)}=\frac{2}{\pi \hbar^{2} v^{2}} \int\left|\varepsilon_{n}(q)\right| q d q \\
=\frac{n_{0} U^{2}}{2 \gamma_{1}}\left[x \sqrt{x^{2}-1}+\ln \left(x+\sqrt{x^{2}-1}\right)\right] .
\end{array}
$$

The energy of the electrostatic field (see Fig. 3)

$$
V^{(f)}=\frac{1}{8 \pi}\left(d E^{2}+\epsilon_{w} d_{w} E_{w}^{2}\right)
$$

can be written in terms of the carrier concentrations with the help of relations

$$
4 \pi e\left(n_{1}-N_{1}\right)=E \quad \text { and } \quad 4 \pi e(n-N)=\epsilon_{w} E_{w},
$$

where $\epsilon_{w}$ is the dielectric constant of the wafer, $N_{1}$ and $N_{2}$ are concentrations of the acceptor or donor impurities on the left and right layers, correspondingly, whereas the total dopant concentration on the bilayer is $N=N_{1}+$ $N_{2}$. All these numbers are supposed to be positive or negative for the electron or hole doping correspondingly. Let us emphasize that the dielectric constant $\epsilon$ of bilayer graphene depends on the substrate. For simplicity, we put $\epsilon=1$ in the definition (14).

We seek the minimum of the total energy

$$
V^{(f)}+V^{(c)}+\lambda\left(e V_{g}+e d E+e d_{w} E_{w}\right)
$$

as a function of $U, \mu$, and the Lagrange multiplier $\lambda$. Differentiation with respect to $\lambda$ gives the gate voltage 
constraint (12). Minimization with respect to $U$ and $x$ gives

$$
\begin{array}{r}
4 \pi e^{2}\left[n_{1}-N_{1}\right) n_{1 u} d+(n-N) n_{u} d_{w} / \epsilon_{w} \\
+V_{u}^{(c)}+4 \pi e^{2} \lambda\left(n_{1 u} d+n_{u} d_{w} / \epsilon_{w}\right)=0
\end{array}
$$

and the similar equation with a substitution $u \rightarrow x$, where the subscripts $u$ and $x$ note the derivatives with respect to the corresponding variables. The Lagrange multiplier $\lambda$ can be excluded from these two equations. Then, the equation obtained should be expanded in $d / d_{w}$, since the thickness $d$ of the bilayer is much less than the thickness $d_{w}$ of the substrate.

Thus, we obtain the following equation:

$$
4 \pi e^{2} d\left(n_{2}-N_{2}\right)\left(\frac{n_{1 x}}{n_{x}}-\frac{n_{1 u}}{n_{u}}\right)=\frac{V_{x}^{(c)}}{n_{x}}-\frac{V_{u}^{(c)}}{n_{u}} .
$$

Let us emphasize, that this equation is invariant under the simultaneous sign change in $n_{1,2}$ and $N_{1,2}$, that expresses the charge invariance of the problem. At the fixed external doping $N_{1,2}$, the gap on the electron and hole sides of the gate bias is not symmetrical.

The derivatives in Eq. (16) are calculated with the help of Eqs. (9)-(15). As a result, Eq. (16) becomes

$$
2 \frac{\gamma_{1} N_{2}}{U n_{0}}=\sqrt{x^{2}-1} \pm\left\{f(x)+\frac{x f(x)}{\Lambda\left[x f(x)-\sqrt{x^{2}-1}\right]}\right\}(17)
$$

with the function $f(x)=\ln \left(x+\sqrt{x^{2}-1}\right)$ and the dimensionless screening constant

$$
\Lambda=\frac{e^{2} \gamma_{1} d}{(\hbar v)^{2}} .
$$

For the parameters of graphene $d=3.35 \stackrel{\circ}{A}, \gamma_{1}=0.381$ $\mathrm{eV}$, and $v=1.02 \times 10^{8} \mathrm{~cm} / \mathrm{s}$, we get $\Lambda=0.41$.

\section{THE GAP IN UNDOPED AND DOPED BASED BILAYER}

\section{(i) undoped bilayer}

First, let us consider an ideal undoped bilayer with $N_{1}=N_{2}=0$. We get a nonzero solution for $U$, if the right-hand side of Eq. (17) vanishes. This condition is fulfilled only for the sign "-" in Eq. (17), that defines the polarity of the layers [see Eq. (91)]. We obtain solution $x=x_{0}=6.61$. According to Eq. (11), the gap as a function of the carrier concentration takes a very simple form:

$$
2|U / n|=\frac{2 \gamma_{1}}{n_{0} \sqrt{x_{0}^{2}-1}}=1.13 \times 10^{-11} \mathrm{meV} \cdot \mathrm{cm}^{2},
$$

where the right-hand side does not depend at all on the gate bias, but only on the screening constant $\Lambda$. This

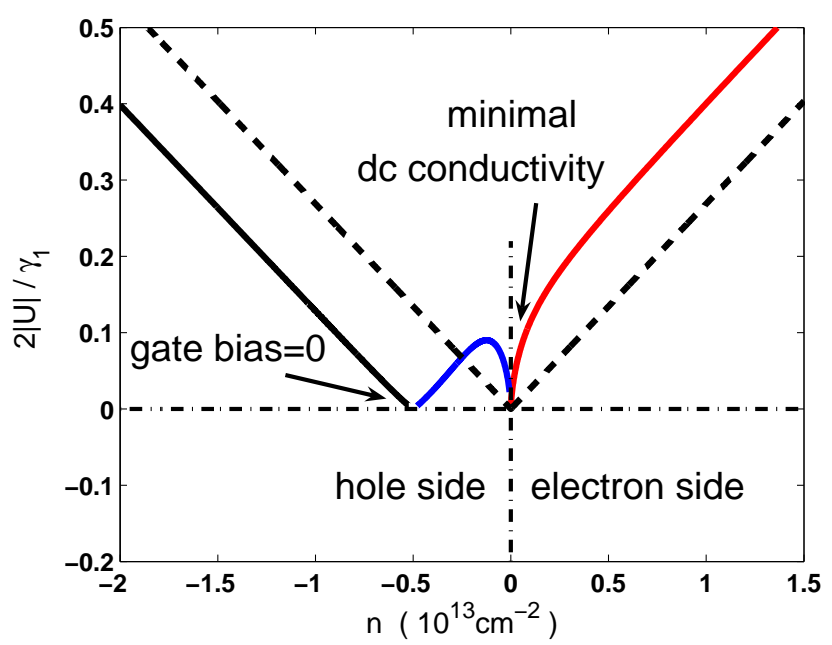

FIG. 4: The gap in units of $\gamma_{1}=0.381 \mathrm{eV}$ versus the carrier concentration in absence of doping (dashed line) and for the hole doping level $N_{2}=-2.5 \times 10^{12} \mathrm{~cm}^{-2}$ (solid line); the positive (negative) values of $n$ correspond to the electron (hole) conductivity. The difference between values of $n$ marked as "gate bias $=0 "$ and "minimal dc conductivity" is $2 N_{2}$.

dependence is shown in Fig. 4 in dashed lines, it is symmetrical on the electron and hole sides.

With the help of Eq. (10), we obtain the chemical potential as a linear function of the carrier concentration

$$
\mu=\frac{\gamma_{1} x_{0}}{n_{0} \sqrt{x_{0}^{2}-1}} n
$$

where $n$ is positive (negative) for the electron (hole) conductivity.

We can compare Eq. (19) with the corresponding result of Ref. [11]:

$$
2|U / n|=\frac{e^{2} d}{2 \epsilon_{0}}\left[1+2 \Lambda \frac{|n|}{n_{0}}+\Lambda \ln \frac{n_{0}}{|n|}\right]^{-1} .
$$

Both equations give approximately the same results at $|n| \simeq 0.1 n_{0} \simeq 10^{12} \mathrm{~cm}^{-2}$. However, contrary to Eq. (19), Eq. (21) contains the carrier concentration in the right-hand side giving rise to the more rapid increase in the gap with $|n| \ll n_{0}$. This increase also contradicts to the DFT calculations [13].

Two reasons can result in the disagreement of our theory with Ref. [11]. First, in Ref. 11], the filled bands are supposed to create the electric field in the bilayer, that is incorrect as explained in the previous section. Second, the minimization should be done with respect two variables $\mu$ and $U$, however, only one of them seems to be exploited in Ref. [11]. 


\section{(ii) doped bilayer}

For the bilayer with the acceptor or donor dopants, Eq. (17) presents a solution $w=2 \gamma_{1} N_{2} / U n_{0}$ as a function of $x$. We obtain, evidently, the small values of $w$ for $x$ close to $x_{0}=6.61$. Since $x_{0} \gg 1$, we can expand the function in the right-hand of Eq. (17) in 1/x. In this region of the relatively large $|U|$, we find again with the help of Eqs. (11) and (17) the linear dependence

$$
\begin{array}{r}
2|U|=\left|n-2 N_{2}\right| \frac{2 \gamma_{1}}{n_{0} x_{0}} \\
=1.13\left|n-2 N_{2}\right| \times 10^{-11} \mathrm{meV} \cdot \mathrm{cm}^{2} .
\end{array}
$$

The value of the carrier concentration $n=2 N_{2}$ corresponds to the zero bias voltage, where $U=0$ (see Fig. 4). Therefore, in contrast with the undoped case, the gap demonstrates the asymmetrical behavior on the electron and hole sides. If the bilayer contains acceptors with concentration $N_{2}$, the gap decreases linearly with the hole concentration and vanishes, when the gate bias is not applied and the hole concentration equals $2 N_{2}$. Starting from this point, the gap increases and, thereafter, becomes again small (equals zero in Fig. 4) at the carrier concentration corresponding to the minimal value of the dc conductivity, where $n=0$. Therefore, the difference $\left(1.56 \times 10^{12} \mathrm{~cm}^{-2}\right.$ in Fig. 4) observed in Refs. 9] and [10] between these two values of carrier concentrations, at the zero bias and at the minimal conductivity, gives directly the donor/acceptor concentration $\left(2 N_{2}\right)$ on the layer close to the substrate. Then, for the gate bias applied in order to increase the electron concentration, the gap is rapidly opening with the electron appearance.

We see, that the asymmetry arises between the electron and hole sides of the gate bias. This asymmetry can simulate a result of the hopping integral $\Delta$ in the electron spectrum [18]. In order to obtain the gap dependence for the case of electron doping, $N_{2}>0$, the reflection transformation $n \rightarrow-n$ has to be made. This case is shown in Fig. 5] where the experimental data from Ref. [10] are displayed.

The gap in the vicinity of the minimal conductivity value could reach indeed a finite value due to several reasons. One of them is the form of the "mexican hat" shown in Fig. 2, Second, the trigonal warping is substantial at low carrier concentrations. Finally, the graphene electron spectrum is unstable with respect to the Coulomb interaction at the low momentum values. For the graphene monolayer, as shown in Ref. [19], the logarithmic corrections appear at the small momentum. In the case of the bilayer, the electron self-energy contains the linear corrections, as can be found using the perturbation theory. The similar linear terms resulting in a nematic order were also obtained in the framework of the renormalization group [20].

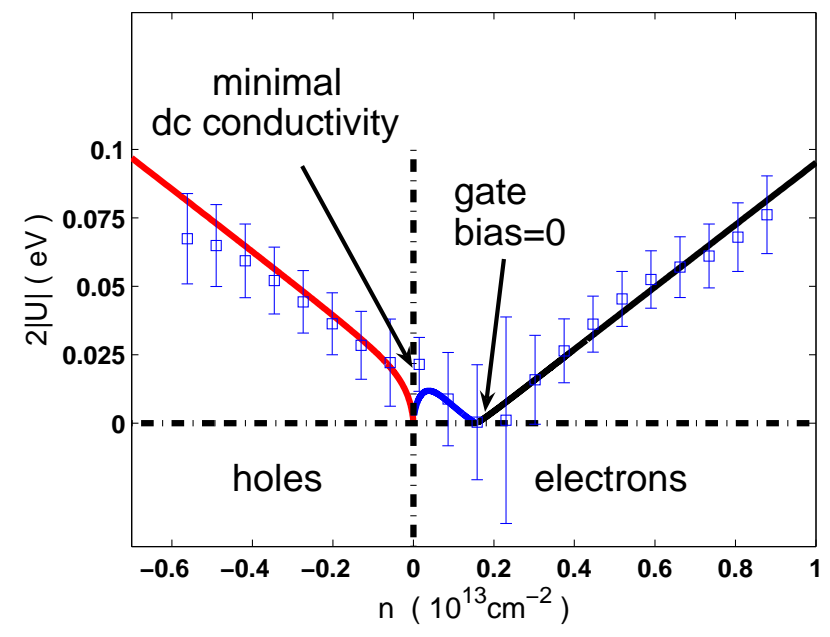

FIG. 5: The gap in eV versus the carrier concentration for the electron doping with the concentration $N_{2}=0.78 \times 10^{12} \mathrm{~cm}^{-2}$ (our theory); the positive (negative) values of $n$ correspond to the electron (hole) conductivity; squares are experimental data [10].

\section{CONCLUSION}

The gap opening in the gated graphene bilayer has an intriguing behavior as a function of carrier concentration. In the presence of the external doping charge, i. e. donors or acceptors, this function is asymmetric on the hole and electron sides of the gate bias and it is the linear function only for the large gate bias. A difference between two values of carrier concentrations, i. e. at the zero bias and at the minimal conductivity, gives directly the sign and concentration of the charged dopants on the bilayer.

I thank A.M. Duygaev and Y.N. Ovchinnikov for helpful discussions and A.B. Kuzmenko for providing his experimental data prior to publication. This work was supported by the Russian Foundation for Basic Research (grant No. 07-02-00571). The author is grateful to the Max Planck Institute for the Physics of Complex Systems for hospitality in Dresden.

[1] E. McCann, V.I. Fal'ko, Phys. Rev. Lett. 96, 086805 (2006).

[2] C.L. Lu, C.P. Chang, Y.C. Huang, R.B. Chen, and M.L. Lin, Phys. Rev. B 73, 144427 (2006).

[3] T. Ohta, A. Bostwick, T. Seyller, K. Horn, and E. Rotenberg, Science 313, 951 (2006).

[4] L.M. Zhang, Z.Q. Li, D.N. Basov, M.M. Foger, Z. Hao, and M.C. Martin, Phys. Rev. B 78, 235408 (2008).

[5] A.B. Kuzmenko, E. van Heumen, D. van der Marel, P. Lerch, P. Blake, K.S. Novoselov, A.K. Geim, Phys. Rev. B 79, 115441 (2009).

[6] Z.Q. Li, E.A. Henriksen, Z. Jiang, Z. Hao, M.C. Martin, 
P. Kim, H.L. Stormer, and D.N. Basov, Phys. Rev. Lett. 102, 037403 (2009).

[7] E.V. Castro, K.S. Novoselov. S.V. Morozov, N.M.R. Peres, J.M.B. Lopes dos Santos, Johan Nilsson, F. Guinea, A.K. Geim, and A.H. Castro Neto, Phys. Rev. Lett. 99, 216802 (2007).

[8] E.J. Nicol, J.P. Carbotte, Phys. Rev. B 77, 155409 (2008).

[9] K.F. Mak, C.H. Lui, J. Shan, and T.F. Heinz, Phys. Rev. Lett. 102, 256405 (2009).

[10] A.B. Kuzmenko, I. Crassee, D. van der Marel, P. Blak, and K.S. Novoselov, arXiv:0908.0672 (2009).

[11] E. McCann, Phys. Rev. B 74, 161403(R) (2006).

[12] E. McCann, D.S.L. Abergel, V.I. Fal'ko, Sol. St. Comm. 143, 110 (2007).

[13] P. Gava, M. Lazzeri, A.M. Saitta, and F. Mauri, Phys.
Rev. B 79, 165431 (2009).

[14] J.C. Slonchewski and P.R. Weiss, Phys. Rev. 109, 272 (1958).

[15] J.W. McClure, Phys. Rev. 108, 612 (1957).

[16] X.G.J.C. Charlier and J.P. Michenaud, Phys.Rev. B 43, 4579 (1982).

[17] B. Partoens, F.M. Peeters, Phys. Rev. B 74, 075404 (2006).

[18] E.V. Castro, K.S. Novoselov. S.V. Morozov, N.M.R. Peres, J.M.B. Lopes dos Santos, Johan Nilsson, F. Guinea, A.K. Geim, and A.H. Castro Neto, arXiv:0807.3348 (2008).

[19] E.G. Mishchenko, Phys. Rev. Lett. 98, 216801 (2007).

[20] O. Vafek and K. Yang, arXiv:0906.2483 (2009). 